\title{
Role of bile acids in the prediction of hepatocellular carcinoma in HCV-induced liver cirrhosis
}

\author{
Ashraf Khalil $^{1 *}$ (D), Azza Elsheashaey ${ }^{1,2}$, Eman Abdelsameea ${ }^{3}$, Manar Obada ${ }^{1}$, F. F. Mohamed Bayomy ${ }^{1,2}$ and \\ Hala El-Said ${ }^{1}$
}

\begin{abstract}
Background: Bile acids are essential organic molecules synthesized from cholesterol in the liver and regarded as indicators of hepatobiliary impairment; however, their role in the pathogenesis of hepatocellular carcinoma (HCC) is still unclear. The study aimed to examine the feasibility of bile acids in distinguishing HCC from post hepatitis $C$ virus liver cirrhosis. A UPLC/MS was used to measure 14 bile acids in patients with noncirrhotic HCV disease $(n=50)$, cirrhotic HCV disease $(n=50)$, hepatocellular carcinoma $(n=50)$, and control group $(n=50)$.

Results: The progression of liver cirrhosis to HCC was associated with a significant increase in serum bile acids compared to the normal or the noncirrhotic HCV disease $(p<0.05)$. The fold changes in bile acids concentrations showed a trend that HCC > cirrhotic HCV disease > noncirrhotic HCV disease. Four conjugated acids GCA, GCDCA, GUDCA, and TCDCA steadily increased across the different groups. ROC curves analysis revealed that these bile acids discriminated noncirrhotic liver patients from HCC (AUC 0.850-0.963), with a weaker potential to distinguish chronic liver cirrhosis from HCC (AUC 0.414-0.638).

Conclusion: The level of serum bile acid was associated primarily with liver cirrhosis, with little value in predicting the progress of chronic liver cirrhotic disease into hepatocellular carcinoma.
\end{abstract}

Keywords: Cirrhosis, Hepatocellular carcinoma (HCC), Liquid chromatography-mass spectrometry; Metabolic profiling

\section{Background}

Bile acids constitute more than 20 molecules synthesized by the liver as primary bile acids cholic acid (CA) and chenodeoxycholic acid (CDCA) then modified by intestinal bacteria into secondary bile acids deoxycholic acid (DCA), lithocholic acid (LCA), and ursodeoxycholic acid (UDCA). Conjugation of the bile acids through the enterohepatic circulation results in more water-soluble bile acids and thus protecting against hepatic cellular damage from the toxic hydrophobic bile acids, which can induce oxidative stress and cell death signaling [1].

\footnotetext{
*Correspondence: ashkalil2010@gmail.com

'Department of Biochemistry and Molecular Diagnostics, National Liver

Institute, Menoufia University, Shebeen El kom, Egypt

Full list of author information is available at the end of the article
}

Numerous studies related liver cirrhosis to the changes in bile acid metabolism, and high serum bile acids can distinguish liver cirrhosis with higher sensitivity than the traditional liver function tests [2-4]. Bile acids metabolism has a role in cellular processes related to carcinogenesis, e.g., elevated intracellular concentrations of bile acids were associated with oxidative stress and DNA damage both in adult and fetal liver $[5,6]$. Bile acid may trigger apoptosis by directly activating the Fas death receptor or through mitochondrial dysfunction secondary to oxidative damage. Therefore, the disturbance in bile acid metabolism could be an early clue in the development of HCC, which is aggressive cancer, with around 90\% of cases developing from pre-existing liver cirrhosis [7-10]. Early detection of HCC remains a challenge as it is typically diagnosed at advanced stages [11, 12], and 
there are no clinically approved alternatives to alphafetoprotein (AFP) that could form a noninvasive test for early detection of HCC. AFP had a low sensitivity as $40 \%$ of HCC patients have normal AFP levels, and only $20 \%$ of patients with early HCC have elevated AFP levels [13]. Des-gamma-carboxyprothrombin and lectin-bound AFP (AFP-L3), glypican-3, Osteopontin, or high c-met expression were hypothesized as alternatives, but their sensitivity for HCC remains unsatisfactory especially, for small lesions [14-18]. In this study, a metabolomics approach applying, ultra-performance liquid chromatography coupled with mass spectrometry was conducted to characterize 14 bile acids profiles in the serum of patients with post $\mathrm{HCV}$ noncirrhotic liver disease, in $\mathrm{HCV}$ cirrhotic liver disease, and post HCV complicating HCC patients, as potential markers for HCC.

\section{Patients}

The study was carried out in the Departments of Biochemistry and Molecular diagnostics of the National Liver Institute hospital, Menouffia University, Egypt, from October 2017 to August 2018 and included three groups. The HCV-noncirrhotic liver disease (NCLD) group $(n=50)$ enrolled patients with a documented HCV infection for $\geq 6$ months without any clinical or imaging (ultrasound and fibro scan) evidence of liver cirrhosis. The post hepatitis $\mathrm{C}$ cirrhotic liver disease (CLD) group $(n=50)$ enrolled patients with liver cirrhosis secondary to previous HCV infection. The post hepatitis C liver cirrhosis complicated with HCC group $(n=50)$ enrolled patients whose HCC developed on the existing liver cirrhosis complicating chronic HCV infection. The NHC group $(n=50)$ enrolled normal, healthy subjects, matching the age and the gender of the other groups with no clinical, laboratory, or imaging sign of liver cirrhosis or focal hepatic lesions. NHC subjects were also free from any other cancers, diabetes mellitus, and obesity and were abstinent from drug abuse and alcohol consumption.

\section{Inclusion criteria}

Liver cirrhosis based on the established clinical findings, liver function tests, and positive serological tests (anti-HCV antibody and HCV- RNA PCR tests), fibro scan $\geq 14.5 \mathrm{kPa}$, and liver ultrasound confirming the characteristic echogenic pattern of liver cirrhosis. The noncirrhotic patients had a history of $\mathrm{HCV}$ infection $\geq 6$ months with positive serological tests without evidence of liver cirrhosis by fibro scan and ultrasound examination of the liver [19]. HCC diagnosis by imaging consisting of single or multiple focal hepatic lesion(s) associated with elevated serum AFP > $200 \mathrm{ng} /$ $\mathrm{ml}$ and or detection of HCC by histological examination of the liver biopsy. The study used the standard
Child-Pugh classification in CLD and HCC groups [20] and the Barcelona Clinic Liver Cancer (BCLC) staging system to stage HCC [21]. No history of alcohol intake or illicit drug abuse in all patients enrolled in the study.

\section{Exclusion criteria}

Patients having both HCV and HBV infection, chronic cholestasis, and obstructive gall bladder diseases, liver disease associated with severe renal or systemic diseases as cardiovascular, DM, and obesity were excluded.

\section{Ethical considerations}

The research ethics committees of the National Liver Institute (IRB00003425), Menouffia University, approved the research proposal and the protocols to comply with national research guidelines. Patients provided informed written consent for the use of tissue for research purposes.

\section{Methods \\ Chemicals and reagents}

HPLC grade methanol, acetonitrile, and formic acid were purchased from Fisher Scientific (Daytona plus, Randox laboratories limited, UK). Bile acid standards are as follows: cholic acids (CA), chenodeoxycholic acid CDCA, deoxycholic acid DCA, lithocholic acid LCA, ursodeoxycholic acid UDCA, glycocholic acid (GCA), glycochenodeoxycholic acid (GCDCA), glycodeoxycholic acid (GDCA), glycoursodeoxycholic acid (GUDCA), taurocholic acid (TCA), taurochenodeoxycholic acid (TCDCA), taurodeoxycholic acid (TDCA), tauroursodeoxycholic acid (TUDCA), and taurolithocholic acid (TLCA) from Sigma Chemical Sigma-Aldrich (Sysmex KX-21, Sysmex Inc., Japan). HPLC grade water from Millipore pure water purification system (Diamond TII, USA).

\section{Serum sample collection}

Five milliliters of blood were collected from patient and control subjects after overnight fasting about $8-12 \mathrm{~h}$, under a sterile venipuncture, and the extracted serum was stored at $-80^{\circ} \mathrm{C}$ until UPLC analysis. Blood chemistry was measured by an automatic biochemical analyzer (Bachman Ltd, London, UK).

\section{Serum sample preparation and bile acid detection}

Serum bile acids were prepared for UPLC/MS/MS as described in [22]. One hundred microliters of the serum sample mixed with $400 \mu \mathrm{l}$ of ice-cold methanol were centrifuged at $12500 \mathrm{rpm}$ for $20 \mathrm{~min}$, and then $50 \mu \mathrm{l}$ of the supernatant was added to $100 \mu \mathrm{l}$ of the mobile phase A $(0.001 \%$ formic acid) where $5 \mu \mathrm{l}$ was injected into a C18 column $(1.7 \mu \mathrm{m}, 100 \mathrm{~mm} \times 2.1 \mathrm{~mm}$ internal 
dimensions) of the ultra-performance liquid chromatography at $50^{\circ} \mathrm{C}$ (Waters ACQUITY, Milford, MA). The mass spectrometer had an electrospray source operated in the negative ion mode using the multiple reactions monitoring (MRM). Each bile acid was eluted by gradient at a flow rate of $0.5 \mathrm{ml} / \mathrm{min}$, for 2 min with $80 \%$ mobile phase A (0.001 formic acid in water) and 20\% mobile phase $B$ (acetonitrile), then with a linear gradient of $20 \%$ mobile phase B over 5 min followed by mobile phase B at (80\%) for $8 \mathrm{~min}$. At the end of each cycle, the column was equilibrated with $80 \%$ mobile phase A for 2 min. UPLC-MS raw data obtained with MRM mode were analyzed using Target Lynx application manager version 4.1 (Waters Corp., Milford, MA) to get the quantitative concentration of each bile acid.

\section{Calibration curves and method assessment}

Seven serially diluted standard calibration points, ranging from 0.125 to $20 \mu \mathrm{mol} / \mathrm{l}$, and three quality control
(QC) standards points 0.2 , 2, and $20 \mu \mathrm{mol} / \mathrm{l}$ were prepared from the 14 bile acids mixture and the QC standard in charcoal-stripped serum. Calibrators and QC standards underwent the sample preparation process described before and were used to calibrate the machine. Calibration curves confirmed that bile acids had a linear response, with a coefficient of determination (R2) $\geq 0.99$. The recovery was evaluated by comparing the mean detector response of the extracted QC samples at 0.2, 2, and $20 \mu \mathrm{mol} / \mathrm{l}$ in triplicates to the mean detector response of the post-extracted serum blanks spiked at equal concentrations. The accuracy and precision were checked regularly before any assay using three replicates of freshly prepared QC standard samples at $0.2,2$, and $20 \mu \mathrm{mol} / \mathrm{l}$. Accuracy was calculated from the formula \% relative error (RE) [\% (measured-theoretical)/theoretical concentration]. Precision was calculated from the formula relative standard deviation $(\% \mathrm{RSD}=\%$ standard deviation/mean). The developed UPLC-MS/MS assay

Table 1 Demographic, clinical, and hematological parameter of the enrolled groups

\begin{tabular}{|c|c|c|c|c|}
\hline & $\mathrm{NHC}$ & NCLD & CLD & $\mathrm{HCC}$ \\
\hline Age, mean (range) & $45(34-73)$ & $46(36-69)^{\mathrm{NS}}$ & $46(37-70)^{N S}$ & $46(37-69)^{\mathrm{NS}}$ \\
\hline $\mathrm{BMI}\left(\mathrm{kg} / \mathrm{m}^{2}\right)$ & $23.5 \pm 0.4$ & $23.6 \pm 0.3^{N S}$ & $22.9 \pm 0.3^{N S}$ & $23.4 \pm 0.3^{\mathrm{NS}}$ \\
\hline \multicolumn{5}{|l|}{ Sex } \\
\hline Male & $25(50 \%)$ & $19(38 \%)$ & $29(58 \%)$ & $15(30 \%)$ \\
\hline Female & $25(50 \%)$ & $31(62 \%)$ & $21(42 \%)$ & $35(70 \%)$ \\
\hline AFP ng/ml, median (IQR) & $1.7(0.8)$ & $2.3(1.2)^{\mathrm{NS}}$ & $3.7(4.4)^{*}$ & $68.4(877)^{*}$ \\
\hline Child-Pugh class, A/B/C & & & $38(76 \%) / 8(16 \%) / 4(8 \%)$ & $18(36 \%) / 17(34 \%) / 15(30 \%)$ \\
\hline HFL, single/multiple & & & & 19 (38\%)/31 (62\%) \\
\hline Metastasis, No/Yes & & & & $46(92 \%) / 4(8 \%)$ \\
\hline Lymph node, No/yes & & & & $43(86 \%) / 7(14 \%)$ \\
\hline PV invasion, No/yes & & & & $50(100 \%) / 0(0 \%)$ \\
\hline Barcelona, HCC stage A/B/C & & & & $36(72 \%) / 10(20 \%) / 4(8 \%)$ \\
\hline AST (IU/L) & $20.7 \pm 6.5$ & $39.8 \pm 34.4^{*}$ & $56.6 \pm 35^{*}$ & $55.4 \pm 29.2^{*}$ \\
\hline ALT (IU/L) & $20.2 \pm 8.6$ & $41.3 \pm 36.7^{*}$ & $41.9 \pm 44.2^{*}$ & $31.9 \pm 18.5^{*}$ \\
\hline GGT (IU/ml) & $23 \pm 13$ & $35 \pm 22^{*}$ & $71 \pm 53^{*}$ & $73 \pm 61^{*}$ \\
\hline ALP (IU/ml) & $60.3 \pm 22.6$ & $74.5 \pm 47^{\mathrm{NS}}$ & $113.5 \pm 57^{*}$ & $117.2 \pm 49.2^{*}$ \\
\hline TBil (mg/dl) & $0.5 \pm 0.2$ & $0.6 \pm 0.3^{*}$ & $1.5 \pm 1.6^{*}$ & $1.5 \pm 1.2^{*}$ \\
\hline DBil (mg/dl) & $0.2 \pm 0.1$ & $0.2 \pm 0.1^{\mathrm{NS}}$ & $1.2 \pm 1.7^{*}$ & $0.8 \pm 0.7^{*}$ \\
\hline ALB (g/dl) & $4 \pm 0.2$ & $4.7 \pm 0.3$ & $3.5 \pm 0.8^{*}$ & $3.3 \pm 0.7^{*}$ \\
\hline TP (mg/dl) & $7 \pm 0.9$ & $8 \pm 0.4^{\mathrm{NS}}$ & $8 \pm 0.7^{\mathrm{NS}}$ & $7 \pm 0.7^{*}$ \\
\hline $\mathrm{Hb}(\mathrm{g} / \mathrm{l})$ & $134 \pm 12$ & $131 \pm 15^{\text {NS }}$ & $119.8 \pm 20^{*}$ & $120 \pm 20^{*}$ \\
\hline Platelets x $\left(10^{9} / \mathrm{l}\right)$ & $290.9 \pm 72.2$ & $259 \pm 91$ NS & $132.3 \pm 63.8^{*}$ & $128.7 \pm 63.1^{*}$ \\
\hline WBCs $\times\left(10^{9} / \mathrm{I}\right)$ & $7.3 \pm 1.4$ & $6.9 \pm 1.5^{\mathrm{NS}}$ & $5.4 \pm 2.3^{*}$ & $5 \pm 1.9^{*}$ \\
\hline
\end{tabular}

NHC normal healthy control, CLD cirrhotic liver diseases, HCC hepatocellular carcinoma. $N=50$ for each group, value $=$ mean \pm standard deviation, median; $I Q R$ interquartile range, ${ }^{*} P$ value $<0.05$ indicates significance when NHC compared to NCLD, CLD, and HCC. ${ }^{\mathrm{NS}} P$ value $>0.05$ indicates non significance when NHC compared to NCLD, CLD, and HCC. BMI body mass index, HFL hepatic focal lesion, PV portal vein, AST aspartate transaminase, ALT alanine transaminase, GGT gamma-glutamyl transferase, $A L P$ alkaline phosphatase, TBil total bilirubin, $D B i L$ direct bilirubin, $T P$ total protein, $A l b$ albumin, $H b$ hemoglobin, $W B C s$ white blood cells 
method had the capability of quantitation of all the 14 bile acids included in the study. The assay performance was accurate and precise for bile acid analysis in the human serum [22].

\section{Statistical analysis}

Data were analyzed using SPSS 23 (SPSS Inc., CA, USA). The nonparametric Kruskal Wallis test and the MannWhitney test were used to detect the significance in multiple comparisons. The receiver operating characteristic (ROC) curve was used to assess the ability of bile acids to distinguish healthy subjects from patients with liver diseases. AUC $\geq 0.8$ was considered as a significant test result to discriminate between two groups. Youden's index or $J$ obtained from equation $J=[$ (sensitivity + specificity) - 1] was applied to select a cutoff, where the sensitivity and the specificity are maximal. Pearson correlation analysis was used to assess relationships between the serum bile acids and AFP. Multivariate analysis was used to detect the predictive potential of bile acids to HCC $[23,24]$.

\section{Results}

\section{Clinical characteristics and laboratory parameters of study} groups

Table 1 presents the anthropometric and clinical parameters of the NCLD, CLD, and HCC groups. Patients were matched by age, gender, and body mass index (BMI) to control the biological and lifestyle confounders. These parameters did not show any significant differences across groups, all $P>0.05$. In the NCLD group, all 50 patients had a well-compensated liver function. In the CLD group, the patients were Child-Pugh A $(n=38)$, Child-Pugh B $(n=8)$, and Child-Pugh C $(n=$ 4). The HCC patients were Child-Pugh A $(n=18)$, Child-Pugh B $(n=17)$, and Child-Pugh C $(n=15)$. According to Barcelona staging system HCC group were stage A $(n=13)$, stage B $(n=10)$, and stage C $(n=7)$. HCC patients had either single focal lesion $(n=19)$ or multiple focal lesions $(n=31)$, lymph node involvement $(n=7)$, or distant metastasis $(n=4)$, but none had portal vein invasion. The laboratory parameters showed that CLD and HCC groups had a significant increase in AST, ALT, TBil, DBil, GGT, ALP, and AFP with a significant decrease in total protein $\mathrm{TP}, \mathrm{Alb}, \mathrm{Hb}, \mathrm{WBCs}$, and platelets relative to the control group, (all $P<0.05$ ). However, there was no statistically significant difference between the NCLD group and the NHC regarding DBil, TP, Hb, and WBCs (all $P>0.05$ ). The levels of DBil, GGT, and ALP increased while the level of Alb, TP, Hb, and platelets decreased in cirrhotic patients compared to NCLD or NHC (all $P<0.05$ ).

\section{Serum bile acid patterns in different stages of liver impairments}

Table 2 presents the comparison of serum bile acids across the four groups. The progress of liver disease was associated with an increase in the serum level of bile acids. The serum bile acids were significantly higher in CLD and HCC groups than either NCLD or NHC groups. Eight bile

Table 2 Serum bile acids and fold changes in the studied groups

\begin{tabular}{|c|c|c|c|c|c|c|c|}
\hline $\mathrm{BA}$ & $\mathrm{NHC}$ & NCLD & CLD & $\mathrm{HCC}$ & F, NCLD & F, CLD & $\mathrm{F}, \mathrm{HCC}$ \\
\hline CA & $0.20 \pm 0.3$ & $0.6 \pm 1.7^{\mathrm{NS}}$ & $0.5 \pm 0.86^{*}$ & $1.7 \pm 4^{*}$ & $3^{*}$ & $3^{*}$ & $9^{*}$ \\
\hline CDCA & $0.38 \pm 0.51$ & $0.73 \pm 1.2^{\mathrm{NS}}$ & $1.45 \pm 1.91^{*}$ & $5.1 \pm 14^{*}$ & $2^{*}$ & $4^{*}$ & $13^{*}$ \\
\hline DCA & $0.15 \pm 0.16$ & $0.19 \pm 0.11^{*}$ & $0.31 \pm 0.40^{\mathrm{NS}}$ & $0.25 \pm 0.39^{\mathrm{NS}}$ & 1 & $2^{*}$ & $2^{*}$ \\
\hline LCA & $0.01 \pm 0.03$ & $0.03 \pm 0.05^{*}$ & $0.05 \pm 0.09^{*}$ & $0.11 \pm 0.20^{*}$ & $3^{*}$ & $4^{*}$ & $9^{*}$ \\
\hline UDCA & $0.04 \pm 0.1$ & $0.04 \pm 0.08^{N S}$ & $1.56 \pm 5.01^{*}$ & $1.99 \pm 6^{*}$ & 1 & $38^{*}$ & $48^{*}$ \\
\hline GCA & $0.24 \pm 0.33$ & $0.43 \pm 0.77^{\mathrm{NS}}$ & $3.77 \pm 4.06^{*}$ & $8.4 \pm 13^{*}$ & $2^{*}$ & $16^{*}$ & $35^{*}$ \\
\hline GCDCA & $0.45 \pm 0.66$ & $0.86 \pm 1.04^{*}$ & $7.7 \pm 11.5^{*}$ & $12.5 \pm 17^{*}$ & $2^{*}$ & $17^{*}$ & $29^{*}$ \\
\hline GDCA & $0.24 \pm 0.36$ & $0.26 \pm 0.33^{N S}$ & $1.48 \pm 2.9^{*}$ & $1.72 \pm 7.2^{\mathrm{NS}}$ & 1 & $6^{*}$ & $7^{*}$ \\
\hline GUDCA & $0.15 \pm 0.2$ & $0.07 \pm 0.12^{*}$ & $5.2 \pm 12.9^{*}$ & $9.4 \pm 30^{*}$ & 0.5 & $35^{*}$ & $38^{*}$ \\
\hline TCA & $0.01 \pm 0.05$ & $0.04 \pm 0.09^{*}$ & $1.7 \pm 2.7^{*}$ & $7.05 \pm 16^{*}$ & $3^{*}$ & $113^{*}$ & $473^{*}$ \\
\hline TCDCA & $0.11 \pm 0.18$ & $0.07 \pm 0.15^{\mathrm{NS}}$ & $4.77 \pm 13.8^{*}$ & $9.1 \pm 16^{*}$ & 0.7 & $44^{*}$ & $83^{*}$ \\
\hline TDCA & $0.07 \pm 0.15$ & $0.02 \pm 0.03^{N S}$ & $0.19 \pm 0.36^{\mathrm{NS}}$ & $1.6 \pm 6^{\mathrm{NS}}$ & 0.3 & $3^{*}$ & $24^{*}$ \\
\hline TLCA & $0.006 \pm 0.028$ & $0.001 \pm 0.002^{*}$ & $0.02 \pm 0.07^{*}$ & $0.08 \pm 0.3^{*}$ & 0.3 & $4^{*}$ & $13^{*}$ \\
\hline TUDCA & $0.04 \pm 0.12$ & $0.01 \pm 0.02^{N S}$ & $0.09 \pm 0.37^{\mathrm{NS}}$ & $0.90 \pm 5.17^{\mathrm{NS}}$ & 0.3 & $2^{*}$ & $18^{*}$ \\
\hline
\end{tabular}

NHC normal healthy control, NCLD noncirrhotic liver disease, CLD cirrhotic liver diseases, HCC hepatocellular carcinoma. $N=50$, number of each group, values: mean \pm standard deviation of bile acids $(\mu \mathrm{M} / \mathrm{L}) ; \mathrm{F}$, fold changes relative to NHC. ${ }^{*} P$ value $<0.05$ indicates significance when NHC compared to either $\mathrm{NCLD}$, CLD, or HCC. ${ }^{\mathrm{NS}} P$ value $>0.05$ indicates significance when NHC compared to either NCLD, CLD, or HCC

CA cholic acid, CDCA chenodeoxycholic acid, DCA deoxycholic acid, LCA lithocholic acid, UDCA ursodeoxycholic acid, GCA glycholic acid, GCDCA glycochenodeoxycholic acid, GDCA glycodeoxycholic acid, GUDCA glycoursodeoxycholic acid, TCA taurocholic acid, TCDCA taurochenodeoxycholic acid, TDCA taurodeoxycholic acid, TLCA taurolithocholic acid, TUDCA tauroursodeoxycholic acid 
acids (CA, CDCA, UDCA, TCA, GCA, GUDCA, TCDCA, and GCDCA) were significantly higher in CLD and HCC than in NHC or NCLD (all $P<0.05$ ). The fold change of bile acids relative to the NHC showed a pattern that HCC $>$ CLD > NCLD, and the increase in the fold was mainly prominent in conjugated bile acids.

\section{Serum bile acids as potential marker of chronic liver impairment}

ROC analysis of the 14 serum bile acids evaluated the ability of bile acids to discriminate HCC from liver cirrhosis. Figure 1 displayed the results of the ROC curves of the 14 bile acids and their diagnostic performance. Five conjugated bile acids (GCA, GCDCA, GUDCA, TCA, and TCDCA) had the best diagnostic performance to separate HCC from NHC with AUC ranging from (0.792-0.963, all $p<0.05)$ and to separate HCC from NCLD with AUC ranging from $(0.795-0.966$, all $p<0.05)$. Bile acids did not discriminate HCC from CLD with AUC ranged from (0.414-0.638, all $p>0.05)$. Table 3 summarizes the AUC, sensitivity, and specificity of the 14 bile acids at the cutoff points detected by Youden's index of the ROC curves.

\section{The interaction of the serum bile acids with the clinico- pathological aspect of HCC}

Table 4 presents the correlation between AFP and bile acids and multivariate analysis between bile acids and the clinicopathological parameter of HCC. Correlation analysis showed that among the 14 bile acids, CA, $r=0.285 p$ $<0.001$; LCA, $r=0.126 p<0.033$; TCA, $r=0.117 p<$ 0.048 ; TLCA, $r=0.128 p<0.031$; and TUDCA, $r=0.656$ $p<0.001$ were positively correlated with AFP.

Multivariate analysis of the clinicopathological feature of HCC (number of the focal lesion, lymph node involvement, metastasis, Child-Pugh score, and Barcelona stage of the disease) revealed that TLCA correlated with three of these clinical parameters, namely, lymph node involvement, number of focal lesions, and the Barcelona stage of HCC. GDCA is associated with metastasis. DCA, TDCA, and TLCA correlated with the Barcelona stage. Five bile acids, one primary and four conjugated bile acids (CDCA, GCA, GDCA, GUDCA, and TUDCA), are associated with the Child-Pugh score. Five bile acids (CA, GCDCA, LCA, TCDCA, and UDCA) did not correlate with any of these clinical parameters and were statistically insignificant $(P>0.05)$.

\section{Discussion}

The study characterized the metabolic profile of 14 bile acids associated with different stages of liver diseases complicating chronic $\mathrm{HCV}$ infection in matched groups of patients with NCLD, LCD, and HCC utilizing a metabolomics approach employing ultrahigh-performance liquid chromatography-tandem mass spectrometry. The
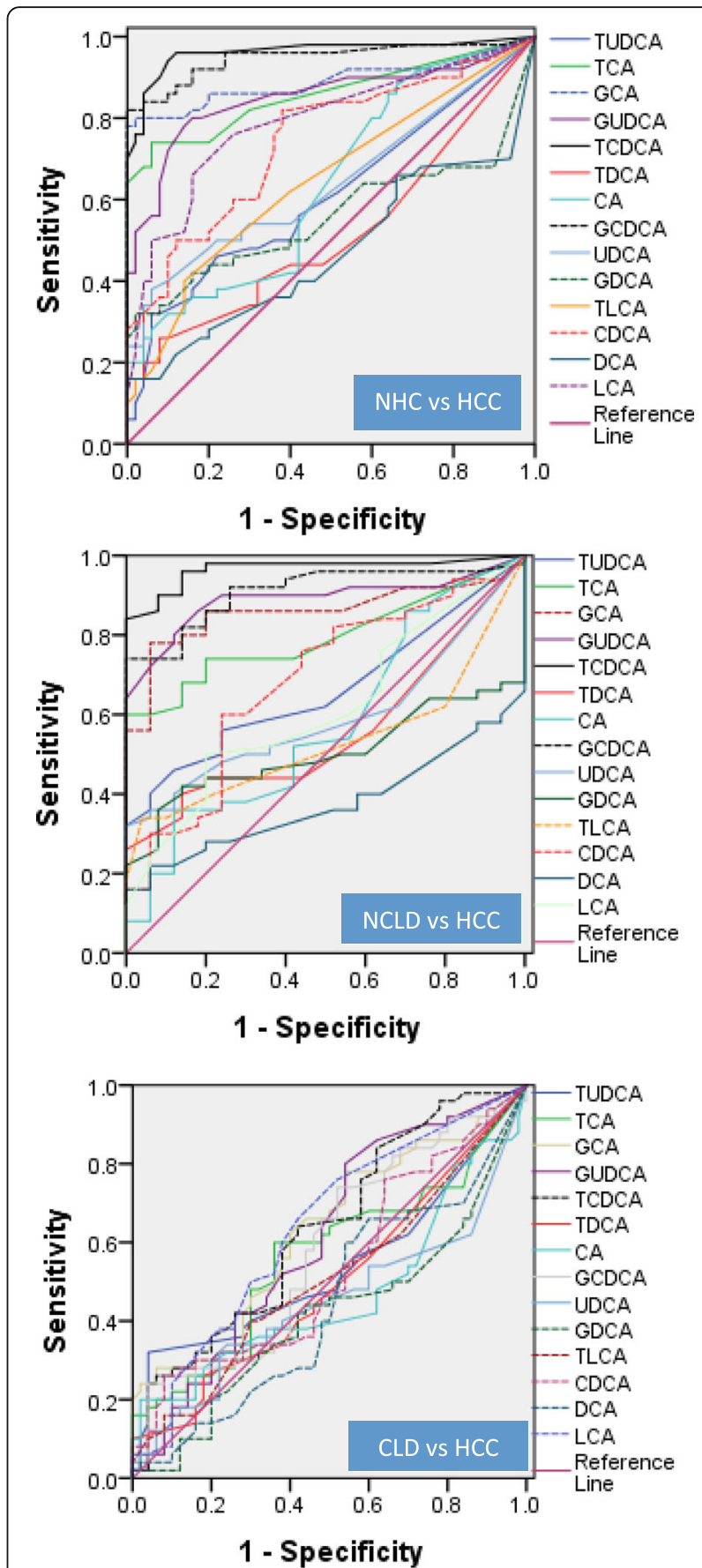

Fig. 1 Receiver operating characteristic curves for the 14 bile acids in the NHC, NCLD, and HCC: the corresponding analytical data obtained from ROC curve analysis including AUC, the cutoff point at the Youden's index, the sensitivity, and the specificity of each bile acid are summarized in Table 3. NHC, normal healthy control; NCLD, noncirrhotic liver disease; CLD, cirrhotic liver diseases; HCC, hepatocellular carcinoma; TUDCA, tauroursodeoxycholic acid; TCA, taurocholic acid; GCA, glycholic acid; GUDCA, glycoursodeoxycholic acid; TCDCA, taurochenodeoxycholic acid; TDCA, taurodeoxycholic acid; CA, cholic acid; GCDCA, glycochenodeoxycholic acid; UDCA, ursodeoxycholic acid; GDCA, glycodeoxycholic acid; TLCA, taurolithocholic acid; CDCA, chenodeoxycholic acid; DCA, deoxycholic acid; LCA, lithocholic acid 
Table 3 Analytical data obtained from ROC curve analysis of 14 Bile acid. The AUC, the cutoff point at the Youden's index, the sensitivity, and the specificity of each bile acid

\begin{tabular}{|c|c|c|c|c|c|c|c|c|c|c|c|c|}
\hline \multirow[t]{2}{*}{ BA } & \multicolumn{4}{|c|}{ NHC vs. HCC } & \multicolumn{4}{|c|}{ NCLD vs. HCC } & \multicolumn{4}{|c|}{ CLD vs HCC } \\
\hline & AUC & Cutoff & Sen $\%$ & Spe $\%$ & AUC & Cutoff & Sen $\%$ & Spe\% & AUC & Cutoff & Sen $\%$ & Spe\% \\
\hline CA & 0.640 & 0.005 & 88 & 34 & 0.577 & 0.35 & 36 & 88 & 0.469 & 2.9 & 20 & 98 \\
\hline CDCA & 0.743 & 0.215 & 82 & 62 & 0.684 & 0.51 & 60 & 76 & 0.532 & 3.35 & 26 & 92 \\
\hline DCA & 0.470 & 0.55 & 16 & 100 & 0.375 & 0.365 & 22 & 94 & 0.442 & 0.045 & 66 & 40 \\
\hline LCA & 0.792 & 0.001 & 76 & 74 & 0.614 & 0.035 & 50 & 80 & 0.638 & 0.001 & 76 & 48 \\
\hline UDCA & 0.643 & 0.12 & 38 & 94 & 0.594 & 0.325 & 32 & 100 & 0.447 & 0.325 & 32 & 78 \\
\hline GCA & 0.889 & 1.55 & 78 & 100 & 0.859 & 1.35 & 78 & 94 & 0.626 & 1.45 & 78 & 46 \\
\hline GCDCA & 0.948 & 1.705 & 84 & 96 & 0.910 & 3.15 & 74 & 100 & 0.584 & 3.1 & 74 & 48 \\
\hline GDCA & 0.558 & 1.015 & 32 & 98 & 0.502 & 0.405 & 42 & 86 & 0.414 & 32.2 & 2 & 100 \\
\hline GUDCA & 0.850 & 0.22 & 80 & 84 & 0.891 & 0.15 & 86 & 82 & 0.612 & 0.215 & 80 & 46 \\
\hline TCA & 0.859 & 0.025 & 74 & 94 & 0.795 & 0.475 & 60 & 100 & 0.563 & 0.435 & 60 & 64 \\
\hline TCDCA & 0.963 & 0.21 & 96 & 88 & 0.966 & 0.55 & 84 & 100 & 0.635 & 0.55 & 84 & 38 \\
\hline TDCA & 0.516 & 0.375 & 20 & 96 & 0.559 & 0.11 & 26 & 100 & 0.496 & 1.025 & 10 & 100 \\
\hline TLCA & 0.648 & 0.002 & 40 & 86 & 0.533 & 0.007 & 34 & 96 & 0.508 & 0.002 & 40 & 70 \\
\hline TUDCA & 0.611 & 0.125 & 32 & 94 & 0.662 & 0.055 & 46 & 88 & 0.536 & 0.115 & 32 & 96 \\
\hline
\end{tabular}

NHC normal healthy control, NCLD noncirrhotic liver disease, CLD cirrhotic liver diseases, $H C C$ hepatocellular carcinoma, $B A$ bile acid, $N=50$, number of each group, $R O C$ receiver operator characteristic, $A \cup C$ Area under curve, Sen. sensitivity, Spe. specificity. AUC $>0.8$ indicates significant relation

$C A$ cholic acid, CDCA chenodeoxycholic acid, DCA deoxycholic acid, LCA lithocholic acid, UDCA ursodeoxycholic acid, GCA glycholic acid, GCDCA

glycochenodeoxycholic acid, GDCA glycodeoxycholic acid, GUDCA glycoursodeoxycholic acid, TCA taurocholic acid, TCDCA taurochenodeoxycholic acid, TDCA taurodeoxycholic acid, TLCA taurolithocholic acid, TUDCA tauroursodeoxycholic acid

changes in the serum bile acids level in the noncirrhotic patients compared to healthy controls were trivial, indicating that the liver can handle the insult without compromising the pool of the bile acids. Four conjugated bile acids, namely GCA, GCDCA, GUDCA, and TCDCA, significantly increased in cirrhotic patients compared with noncirrhotic and were consistent with the clinical and biochemical parameters and thus could be observed as biomarkers of the progress of the liver cirrhosis disease.

In agreement with Zhao et al., this study also found an increase in the conjugated bile acids more than the unconjugated bile acids in cirrhotic and HCC patients, suggesting that conjugated bile acids may reflect the progress of the chronic liver cirrhosis to HCC [25]. Abnormal metabolism of bile acids and oxidative stress are early metabolic changes observed during the progression of liver cirrhosis to early stages of $\mathrm{HCC}$ as they can trigger DNA damage and induce apoptosis [26, 27]. An increase in conjugated bile acids has long been recognized in patients with hepatobiliary diseases such as viral hepatitis, cirrhosis, and cholangiocarcinoma [28]. Bile acid conjugation results in less toxic and more water-soluble bile acid types, thus protecting against cellular damage from such toxic compound that triggers oxidative stress and stimulates cell death signaling [22]. Yang et al. found upregulation of bile acids GCDCA, GDCA, and GCA in patients with hepatitis B compared to healthy control s[25]. Yin Wan et al. detected upregulation of
Table 4 Bile acids correlation analysis with AFP and their multivariate analysis with HCC clinical parameters

\begin{tabular}{|c|c|c|c|c|c|c|}
\hline \multirow[t]{2}{*}{ BA } & \multirow{2}{*}{$\begin{array}{l}\text { Pearson } \\
\text { Correlation } \\
\text { AFP }\end{array}$} & \multicolumn{5}{|c|}{ Multivariate analysis, $\mathrm{F}$ test } \\
\hline & & \# FL & LN & Met & CHP-S & B. stage \\
\hline CA & $0.29 *$ & $0.40^{N S}$ & $0.07^{\mathrm{NS}}$ & $1.03^{\mathrm{NS}}$ & $2.39^{\mathrm{NS}}$ & $0.71^{\mathrm{NS}}$ \\
\hline CDCA & 0.01 NS & $0.07^{N S}$ & $0.13^{N S}$ & $1.27^{\mathrm{NS}}$ & $3.21^{*}$ & $0.12^{N S}$ \\
\hline DCA & $-0.04^{N S}$ & $1.82^{\mathrm{NS}}$ & $3.88^{N S}$ & $0.39^{N S}$ & $1.62^{\mathrm{NS}}$ & $4.02^{*}$ \\
\hline LCA & $0.13^{*}$ & $1.40^{\text {NS }}$ & $1.75^{\mathrm{NS}}$ & $1.34^{\mathrm{NS}}$ & $0.46^{\mathrm{NS}}$ & $0.45^{\text {NS }}$ \\
\hline UDCA & -0.02 NS & $1.53^{\mathrm{NS}}$ & $0.16^{N S}$ & 0.91 NS & $2.34^{\mathrm{NS}}$ & 0.03 NS \\
\hline GCA & $0.04^{\text {NS }}$ & $0.76^{\text {NS }}$ & $0.10^{\text {NS }}$ & $0.26^{\mathrm{NS}}$ & $28.15^{*}$ & $0.45^{N S}$ \\
\hline GCDCA & $0.08^{N S}$ & 0.01 NS & 0.00 NS & $0.11^{\mathrm{NS}}$ & 2.38 NS & $0.37^{\mathrm{NS}}$ \\
\hline GDCA & -0.01 NS & 0.29 NS & $0.34^{N S}$ & $5.07^{*}$ & $1.33^{N S}$ & 0.58 \\
\hline GUDCA & $0.06^{\mathrm{NS}}$ & $0.77^{\mathrm{NS}}$ & $0.14^{\mathrm{NS}}$ & $0.48^{N S}$ & $2.67^{*}$ & $0.29^{N S}$ \\
\hline TCA & $0.10^{*}$ & $0.12^{N S}$ & 0.41 NS & 0.31 NS & $14.90^{*}$ & $2.49^{N S}$ \\
\hline TCDCA & $0.07^{\mathrm{NS}}$ & $0.08^{N S}$ & $0.30^{N S}$ & $0.20^{N S}$ & $0.61^{N S}$ & $0.62^{N S}$ \\
\hline TDCA & -0.01 NS & 1.63 NS & 0.02 NS & $0.13^{N S}$ & $0.18^{N S}$ & $18.14^{*}$ \\
\hline TLCA & $0.13^{*}$ & $4.46^{*}$ & $7.14^{*}$ & $1.26^{\mathrm{NS}}$ & $1.38^{N S}$ & $4.44^{*}$ \\
\hline TUDCA & $0.66^{*}$ & $0.00^{\mathrm{NS}}$ & $0.00^{N S}$ & $0.00^{N S}$ & $4.11^{*}$ & $0.00^{N S}$ \\
\hline
\end{tabular}

$B A$ bile acid, $H C C$ hepatocellular carcinoma, $N=50 ; F L$ number of focal lesions, LN lymph node, Met metastasis, CHP-S Child-Pugh Score, B. stage Barcelona stage of $\mathrm{HCC}$, ${ }^{*}$ indicates $P$ value $<0.05$ and presence of a significant

correlation. ${ }^{\text {Ns }}$ indicates $P$ value $>0.05$ and absence of a significant correlation $C A$ cholic acid, CDCA chenodeoxycholic acid, DCA deoxycholic acid, LCA lithocholic acid, UDCA ursodeoxycholic acid, GCA glycholic acid, GCDCA glycochenodeoxycholic acid, GDCA glycodeoxycholic acid, GUDCA glycoursodeoxycholic acid, TCA taurocholic acid, TCDCA

taurochenodeoxycholic acid, TDCA taurodeoxycholic acid, TLCA taurolithocholic acid, TUDCA tauroursodeoxycholic acid 
four bile acids, GCA, GCDCA, TCA, and TCDCA in cirrhotic patients [23].

In the current study, bile acids profiles did not distinguish HCC from liver cirrhosis, although, GCDCA, GCA, GUDCA, and TCDCA tended to be higher in HCC but without evident statistical significant difference. Several metabolomics studies have identified metabolite expression profile differences between HCC and healthy controls [2, 29], however, as HCC is usually present in patients with liver cirrhosis, it is more relevant to consider cirrhotic patients as a control rather than healthy subjects. The current study had the privilege of including both the NCLD and CLD groups to reflect the progress of liver cirrhosis. Fewer studies reported metabolomics profile differences between HCC and liver cirrhosis [30, 31]. Ressom et al. characterized the metabolic changes relating to HCC in patients with liver cirrhosis and found that bile acids reduced in HCC relative to cirrhosis [32]. Xiao et al. detected a downregulation of three bile acids, GCA, GDCA, and GCDCA, in HCC compared to liver cirrhosis [33]. Chen et al. identified four bile acids CA, GCA, DCA, and GCDCA, altered differently in HCC from liver cirrhosis [2]. The interaction of the bile acids with the clinicopathological features of HCC showed that five bile acids, one primary (CDCA) and four conjugated (GCA, GDCA, GUDCA, TUDCA), correlated with the Child-Pugh score with a predominance of the glycoconjugates form of bile acids. Another three bile acids, one primary (DCA) and two taurine-conjugated (TDCA, TLCA), bile acid correlated with the Barcelona stage of the disease. Therefore, the metabolic profile of these bile acids may predict the progress of liver cirrhosis to HCC [34]. As this study lacks the HCC group without cirrhosis, therefore, the effect of the associated background cirrhosis as a confounding factor could not be ignored, and further studies with noncirrhotic HCC are required to confirm these findings. The limitation of the study is as follows: although patients groups were matched by demographic and clinical characteristics to control factors that may confound interpretation of the bile acids data yet, other diseases such as diabetes, obesity, metabolic syndrome, cardiovascular disease, and gastrointestinal microbiota are related to bile acids metabolism. Therefore, the coexisting of these diseases with liver cirrhosis adds layers of complexity to metabolomics profiling of bile acids [35-38]. The primary objective of this work was to examine the disturbance of bile acids in $\mathrm{HCV}$-induced liver cirrhosis complicated by HCC. Further studies integrating HCC metabolomics data and the relationship of serum bile acid to the direct-acting antiviral agents (DAAs) are needed to delineate the complicated relationship with the other diseases that might confound the result.

\section{Conclusion}

This study characterized the metabolic profile of 14 bile acids in serum in patients with post HCV liver dysfunction ranging from non cirrhosis, cirrhosis, and hepatocellular carcinoma using UPLC-MS/MS methods. The level of conjugated bile acids GCA, GCDCA, GUDCA, and TCDCA were consistently higher in HCC than in NCLD and showed a tendency to be higher in HCC than CLD but without evident statistical significant difference. The increase in the serum bile acids level in patients with $\mathrm{HCV}$-induced liver cirrhosis might serve as warring biomarkers for the progress of liver cirrhosis disease but not HCC.

\begin{abstract}
Abbreviations
NHC: Normal healthy control; NCLD: Noncirrhotic liver disease; CLD: Cirrhotic liver diseases; HCC: Hepatocellular carcinoma; CA: Cholic acid;

CDCA: Chenodeoxycholic acid; DCA: Deoxycholic acid; LCA: Lithocholic acid; UDCA: Ursodeoxycholic acid; GCA: Glycholic acid;

GCDCA: Glycochenodeoxycholic acid; GDCA: Glycodeoxycholic acid; GUDCA: Glycoursodeoxycholic acid; TCA: Taurocholic acid;

TCDCA: Taurochenodeoxycholic acid; TDCA: Taurodeoxycholic acid; TLCA: Taurolithocholic acid; TUDCA: Tauroursodeoxycholic acid;

AST: Aspartate transaminase; ALT: Alanine transaminase; GGT: Gammaglutamyl transferase; ALP: Alkaline phosphatase; TBil: Total bilirubin; DBiL: Direct bilirubin; TP: Total protein; Alb: Albumin; Hb: Hemoglobin; WBCs: White blood cells; HBV: Hepatitis B virus; HCC: Hepatocellular carcinoma; HCV: Hepatitis C virus; AASLD: American Association for the Study of Liver Diseases; AFP: Alpha-fetoprotein; BCLC: Barcelona Clinic Liver Cancer; CT: Computed tomography; ESAL: European Association for the Study of the Liver; NMR: Nuclear magnetic resonance; TNM: (T) tumor, (N) nodes, (M), metastases; FL: Focal lesions; LN: Lymph node; Met: Metastasis; CHP: ChildPugh; HFL: Hepatic focal lesion; PV: Portal vein
\end{abstract}

\section{Acknowledgements}

We would like to express our deep appreciation to all departments and personnel of the National Liver Institute, Menoufia University, for their continuous help and support of the research.

\section{Authors' contributions}

A.S: optimized the UPLC/MS/MS method and performed the experiments. E.A: collection of clinical data and gaining ethical approval. M.O: study design and involved in protocol development. H.S: study concept and contributed reagents, materials, and analysis tools. M.B: help in UPLC/MS/MS method. A.K: corresponding author, analyzed the data, wrote and edited the manuscript All authors reviewed and edited the manuscript and approved the final version of the manuscript.

\section{Funding}

The authors declare they do not have any financial disclosure.

\section{Availability of data and materials \\ The datasets used and/or analyzed during the current study are available from the corresponding author on reasonable request.}

\section{Declarations}

Ethics approval and consent to participate

The research ethics committees of the National Liver Institute (IRB00003413), Menouffia University, approved the research proposal and the protocols to comply with national research guidelines. Patients provided informed written consent for the use of tissue for research purposes.

Consent for publication

Obtained from all participants. 


\section{Competing interests}

The authors declare they do not have any conflict of interest.

\section{Author details}

'Department of Biochemistry and Molecular Diagnostics, National Liver Institute, Menoufia University, Shebeen El kom, Egypt. ${ }^{2}$ Department of Zoology, Faculty of Science, Menoufia University, Shebeen El kom, Egypt; Misr University for Science and Technology (MUST), 6th of October City, Giza, Egypt. ${ }^{3}$ Department of Hepatology and Gastroenterology, National Liver Institute, Menoufia University, Shebeen El kom, Egypt.

Received: 22 April 2021 Accepted: 19 August 2021 Published online: 03 September 2021

\section{References}

1. Altekruse SF, McGlynn KA, Reichman ME (2009) Hepatocellular carcinoma incidence, mortality, and survival trends in the United States from 1975 to 2005. J Clin Oncol 27(9):1485-1491. https://doi.org/10.1200/JCO.2008.20.7753

2. Chen $T$ et al (2011) Serum and urine metabolite profiling reveals potential biomarkers of human hepatocellular carcinoma. Mol Cell Proteomics 10(7): M110 004945

3. Greco AV, Mingrone G (1993) Serum bile acid concentrations in mild liver cirrhosis. Clin Chim Acta 221(1-2):183-189. https://doi.org/10.1016/00098981(93)90032-Y

4. Mannes GA et al (1986) Prognostic significance of serum bile acids in cirrhosis. Hepatology 6(1):50-53. https://doi.org/10.1002/hep.1840060110

5. Baptissart M, Vega A, Maqdasy S, Caira F, Baron S, Lobaccaro JM, Volle DH (2012) Bile acids: from digestion to cancers. Biochimie 95(3):504-517. https://doi.org/10.1016/j.biochi.2012.06.022

6. Davila JA, Morgan RO, Richardson PA, du XL, McGlynn KA, el-Serag HB (2010) Use of surveillance for hepatocellular carcinoma among patients with cirrhosis in the United States. Hepatology 52(1):132-141. https://doi.org/10.1 002/hep.23615

7. Moustafa T, Fickert P, Magnes C, Guelly C, Thueringer A, Frank S, Kratky D, Sattler W, Reicher H, Sinner F, Gumhold J, Silbert D, Fauler G, Höfler G, Lass A, Zechner R, Trauner M (2012) Alterations in lipid metabolism mediate inflammation, fibrosis, and proliferation in a mouse model of chronic cholestatic liver injury. Gastroenterology 142(1):140-151 e12. https://doi. org/10.1053/j.gastro.2011.09.051

8. Chiang JYL, Ferrell JM (2018) Bile acid metabolism in liver pathobiology. Gene Expr 18(2):71-87. https://doi.org/10.3727/105221618X15156018385515

9. Barr DC, Hussain HK (2014) MR imaging in cirrhosis and hepatocellular carcinoma. Magn Reson Imaging Clin N Am 22(3):315-335. https://doi.org/1 0.1016/j.mric.2014.04.006

10. van den Bos IC et al (2007) MR imaging of hepatocellular carcinoma: relationship between lesion size and imaging findings, including signal intensity and dynamic enhancement patterns. J Magn Reson Imaging 26(6): 1548-1555. https://doi.org/10.1002/jmri.21046

11. Chayanupatkul M et al (2017) Hepatocellular carcinoma in the absence of cirrhosis in patients with chronic hepatitis B virus infection. J Hepatol 66(2): 355-362

12. El-Serag HB et al (2013) Hepatocellular carcinoma screening practices in the Department of Veterans Affairs: findings from a national facility survey. Dig Dis Sci 58(11):3117-3126. https://doi.org/10.1007/s10620-013-2794-7

13. Zhao ZH, Lai JKL, Qiao L, Fan JG (2019) Role of gut microbial metabolites in nonalcoholic fatty liver disease. J Dig Dis 20(4):181-188. https://doi.org/1 0.1111/1751-2980.12709

14. Kondo $\mathrm{S}$ et al (2013) Clinical impact of c-Met expression and its gene amplification in hepatocellular carcinoma. Int J Clin Oncol 18(2):207-213

15. Khalil A et al (2013) Plasma osteopontin level as a diagnostic marker of hepatocellular carcinoma in patients with radiological evidence of focal hepatic lesions. Tumori 99(1):100-107

16. Taura $\mathrm{N}$ et al (2013) Frequency of elevated biomarkers in patients with cryptogenic hepatocellular carcinoma. Med Sci Monit 19:742-750

17. Hu K-Q, Kyulo NL, Lim N, Elhazin B, Hillebrand DJ, Bock T (2004) Clinical significance of elevated alpha-fetoprotein (AFP) in patients with chronic hepatitis C, but not hepatocellular carcinoma. Am J Gastroenterol 99(5):860865. https://doi.org/10.1111/j.1572-0241.2004.04152.x

18. Tameda M et al (2013) Des-gamma-carboxy prothrombin ratio measured by P-11 and P-16 antibodies is a novel biomarker for hepatocellular carcinoma. Cancer Sci 104(6):725-731
19. Schutte $\mathrm{K}$ et al (2014) Characterization and prognosis of patients with hepatocellular carcinoma (HCC) in the non-cirrhotic liver. BMC Gastroenterol 14(1):117. https://doi.org/10.1186/1471-230X-14-117

20. Pugh RN, Murray-Lyon IM, Dawson JL, Pietroni MC, Williams R (1973) Transection of the oesophagus for bleeding oesophageal varices. Br J Surg 60(8):646-649. https://doi.org/10.1002/bjs.1800600817

21. de Freitas LBR, Longo L, Santos D, Grivicich I, Álvares-da-Silva MR (2019) Hepatocellular carcinoma staging systems: Hong Kong liver cancer vs Barcelona clinic liver cancer in a Western population. World J Hepatol 11(9): 678-688. https://doi.org/10.4254/wjh.v11.i9.678

22. Luo L, Aubrecht J, Li D, Warner RL, Johnson KJ, Kenny J, Colangelo JL (2018) Assessment of serum bile acid profiles as biomarkers of liver injury and liver disease in humans. PLoS One 13(3):e0193824. https://doi.org/10.1371/journa I.pone.0193824

23. Akobeng AK (2007) Understanding diagnostic tests 3: receiver operating characteristic curves. Acta Paediatr 96(5):644-647. https://doi.org/10.1111/j.1 651-2227.2006.00178.x

24. Fischer JE, Bachmann LM, Jaeschke R (2003) A readers' guide to the interpretation of diagnostic test properties: clinical example of sepsis. Intensive Care Med 29(7):1043-1051. https://doi.org/10.1007/s00134-003-1761-8

25. Yang J, Zhao X, Liu X, Wang C, Gao P, Wang J, Li L, Gu J, Yang S, Xu G (2006) High performance liquid chromatography-mass spectrometry for metabonomics: potential biomarkers for acute deterioration of liver function in chronic hepatitis B. J Proteome Res 5(3):554-561. https://doi.org/10.1021/ pr050364w

26. Agosti P, Sabba C, Mazzocca A (2018) Emerging metabolic risk factors in hepatocellular carcinoma and their influence on the liver microenvironment. Biochim Biophys Acta Mol Basis Dis 1864(2):607-617. https://doi.org/10.1016/j.bbadis.2017.11.026

27. Gowda GA (2010) Human bile as a rich source of biomarkers for hepatopancreatobiliary cancers. Biomark Med 4(2):299-314. https://doi.org/1 0.2217/bmm.10.6

28. Neale G, Lewis B, Weaver $V$, Panveliwalla D (1971) Serum bile acids in liver disease. Gut 12(2):145-152. https://doi.org/10.1136/gut.12.2.145

29. Patterson AD, Maurhofer O, Beyoğlu D, Lanz C, Krausz KW, Pabst T, Gonzalez FJ, Dufour JF, Idle JR (2011) Aberrant lipid metabolism in hepatocellular carcinoma revealed by plasma metabolomics and lipid profiling. Cancer Res 71(21):6590-6600. https://doi.org/10.1158/0008-5472.CAN-11-0885

30. Wang B, Chen D, Chen Y, Hu Z, Cao M, Xie Q, Chen Y, Xu J, Zheng S, Li L (2012) Metabonomic profiles discriminate hepatocellular carcinoma from liver cirrhosis by ultraperformance liquid chromatography-mass spectrometry. J Proteome Res 11(2): 1217-1227. https:/doi.org/10.1021/pr2009252

31. Zhou L, Ding L, Yin P, Lu X, Wang X, Niu J, Gao P, Xu G (2012) Serum metabolic profiling study of hepatocellular carcinoma infected with hepatitis B or hepatitis $C$ virus by using liquid chromatography-mass spectrometry. J Proteome Res 11(11): 5433-5442. https://doi.org/10.1021/pr300683a

32. Ressom HW et al (2012) Utilization of metabolomics to identify serum biomarkers for hepatocellular carcinoma in patients with liver cirrhosis. Anal Chim Acta 743:90-100

33. Xiao JF, Varghese RS, Zhou B, Nezami Ranjbar MR, Zhao Y, Tsai TH, di Poto C, Wang J, Goerlitz D, Luo Y, Cheema AK, Sarhan N, Soliman H, Tadesse MG, Ziada DH, Ressom HW (2012) LC-MS based serum metabolomics for identification of hepatocellular carcinoma biomarkers in Egyptian cohort. J Proteome Res 11(12):5914-5923. https://doi.org/10.1021/pr300673x

34. Kimhofer T, Fye H, Taylor-Robinson S, Thursz M, Holmes E (2015) Proteomic and metabonomic biomarkers for hepatocellular carcinoma: a comprehensive review. Br J Cancer 112(7):1141-1156. https://doi.org/10.103 8/bjc.2015.38

35. Hrncir T, Hrncirova L, Kverka M, Tlaskalova-Hogenova H (2019) The role of gut microbiota in intestinal and liver diseases. Lab Anim 53(3):271-280. https://doi.org/10.1177/0023677218818605

36. Kolodziejczyk AA et al (2019) The role of the microbiome in NAFLD and NASH. EMBO Mol Med 11(2):e9302. https://doi.org/10.15252/emmm.201809302

37. Tang WW, Hazen SL (2016) Dietary metabolism, gut microbiota and acute heart failure. Heart 102(11):813-814. https://doi.org/10.1136/heartjnl-2016-309268

38. Zhao ZH et al (2019) Role of gut microbial metabolites in nonalcoholic fatty liver disease. J Dig Dis 20(4):181-188

\section{Publisher's Note}

Springer Nature remains neutral with regard to jurisdictional claims in published maps and institutional affiliations. 